Pacific

Journal of

Mathematics

\title{
ON THE BOUNDEDNESS OF SINGULAR INTEGRALS
}

Eugene Fabes, Irina Mitrea, and Marius Mitrea 


\title{
ON THE BOUNDEDNESS OF SINGULAR INTEGRALS
}

\author{
Eugene Fabes, Irina Mitrea, and Marius Mitrea
}

\begin{abstract}
We present a simple, elementary proof of the $T(1)$ theorem of G. David and J.-L. Journé, as well as related results, which is based on a Krein type lemma.
\end{abstract}

\section{Introduction.}

Establishing the $L^{2}$-boundedness of singular integral operators is a fundamental problem in harmonic analysis which has always enjoyed center stage. For a broader view of this active area of research see, e.g., [Ca], [CMM], $[\mathbf{M M}],[\mathbf{C D M}],[\mathbf{D J}],[\mathbf{D J S}],[\mathbf{C J S}],[\mathbf{M e}],[\mathbf{C h}],[\mathbf{S t}]$ and the references therein.

The main aim of this note is to indicate yet another way of proving such $L^{2}$ boundedness results which is inspired by an old lemma of M. G. Krein $([\mathbf{K r}])$. Essentially, the latter asserts that if a linear operator $T$ and its formal adjoint $T^{t}$ are bounded on a Banach space $\mathcal{X}$ which is densely and continuously embedded in a Hilbert space $\mathcal{H}$, then $T$ extends to a bounded operator on $\mathcal{H}$. We use this to give a new proof of the celebrated $T(1)$ theorem of David and Journé $([\mathbf{D J}])$ in its full strength.

The actual context in which this lemma applies for a singular integral operator $T$ satisfying the usual set of hypotheses of the $T(1)$ theorem is when $\mathcal{H}=L^{2}$ and $\mathcal{X}=C^{\alpha}$. Of course, one first needs to localize the problem for the latter space to embed properly in the former but, more importantly, one has to ensure boundedness for $T$ at the level of Hölder continuous functions. However, this is essentially well known and requires appropriate cancellations for $T$ which, in turn, are secured by subtracting off paraproducts. Other choices for $\mathcal{X}$ are possible but the one just described yields perhaps the most elementary proof.

This proof is, in principle, quite flexible; in fact, we shall prove a "realvalued" version of the $T(b)$ theorem (cf. [MM], [DJS]), i.e. when the constant 1 is replaced by an arbitrary positive, measurable function $b$ which is bounded away from zero and infinity. The approach can be adapted to spaces of homogeneous type in the sense of $[\mathbf{C o W e}]$ and we believe that the same strategy may also be successful in other cases of interest.

Acknowledgments. We thank Y. Brudnyi, A. McIntosh and M. Christ for several discussions and a suggestion which has been incorporated. 


\section{Statement of theorem.}

Recall that a distribution $k \in \mathcal{D}^{\prime}\left(\mathbb{R}^{n} \times \mathbb{R}^{n}\right)$ is called a standard kernel if its singular support lies on the diagonal and, for some constant $C>0$, satisfies

$$
\begin{aligned}
|k(x, y)| & \leq C|x-y|^{-n}, & & \forall x \neq y, \\
\left|\nabla_{x} k(x, y)\right|+\left|\nabla_{y} k(x, y)\right| & \leq C|x-y|^{-(n+1)} & & \forall x \neq y .
\end{aligned}
$$

Let $\|k\|:=\inf \{C ;(2.1)-(2.2)$ hold $\}$. A linear, continuous operator $T$ : $\mathcal{D}\left(\mathbb{R}^{n}\right) \longrightarrow \mathcal{D}^{\prime}\left(\mathbb{R}^{n}\right)$ is called a singular integral operator (of Calderón-Zygmund type) if its Schwartz distribution kernel is standard. Also, $T$ is said to have the weak boundedness property if there exists a constant $C$ such that

$$
|\langle T \phi, \psi\rangle| \leq C R^{n}\left(\|\phi\|_{\infty}+R\|\nabla \phi\|_{\infty}\right)\left(\|\psi\|_{\infty}+R\|\nabla \psi\|_{\infty}\right)
$$

holds uniformly for $\phi, \psi \in C_{\mathrm{c}}^{\infty}\left(B_{R}(z)\right), R>0, z \in \mathbb{R}^{n}$. Here, and elsewhere, $B_{R}(z)$ stands for the Euclidean ball of radius $R$ centered at $z \in \mathbb{R}^{n}$. We set

$$
\|T\|_{\mathrm{WBP}}:=\inf \{C ; \text { so that }(2.3) \text { holds uniformly }
$$

in the natural parameters\}.

To state the main result, recall that BMO stands for the (John-Nirenberg) space of functions of bounded mean oscillations in $\mathbb{R}^{n}$. Also, a measurable function $b: \mathbb{R}^{n} \rightarrow \mathbb{C}$ is called accretive if there exists $\kappa>0$ (the accretivity constant) such that $\operatorname{Re} b(x) \geq \kappa>0$ for a.e. $x \in \mathbb{R}^{n}$. Finally, $M_{b}$ is the operator of (pointwise) multiplication by $b$.

Theorem 2.1 ([DJ], [MM], [DJS]). Let $b_{1}, b_{2}$ be two essentially bounded, accretive, real-valued functions and let $T$ be a linear continuous operator from $b_{1} C_{c}^{\infty}\left(\mathbb{R}^{n}\right)$ into $\left(b_{2} C_{c}^{\infty}\left(\mathbb{R}^{n}\right)\right)^{\prime}$. Assume that $M_{b_{2}} T M_{b_{1}}$ satisfies the weak boundedness property and that, if $K(x, y)$ stands for its distributional kernel, then $\operatorname{singsupp} K(x, y) \subseteq \operatorname{diag}\left(\mathbb{R}^{n} \times \mathbb{R}^{n}\right)$ and the kernel $k(x, y):=$ $b_{2}(x)^{-1} K(x, y) b_{1}(y)^{-1}$ satisfies $(2.1)-(2.2)$.

Then, if $T\left(b_{1}\right), T^{t}\left(b_{2}\right) \in \mathrm{BMO}$, where $T^{t}$ is the formal adjoint of $T$, it follows that $T$ can be extended to a bounded operator on $L^{2}\left(\mathbb{R}^{n}\right)$ with operator norm controlled by $\|k\|,\left\|T\left(b_{1}\right)\right\|_{\mathrm{BMO}},\left\|T^{t}\left(b_{2}\right)\right\|_{\mathrm{BMO}}$ and $\left\|M_{b_{2}} T M_{b_{1}}\right\|_{\mathrm{WBP}}$.

As usual, $T\left(b_{1}\right)$ is defined as a "non-standard" distribution in view of the fact that it pairs well with elements from $b_{2} C_{\mathrm{c}}^{\infty}\left(\mathbb{R}^{n}\right)$ having vanishing moment. Something similar applies to $T^{t}\left(b_{2}\right)$.

Before commencing the actual proof, we shall record a couple of reduction steps which are going to be important in subsequent arguments. The first one, i.e. the reduction to the case when $T\left(b_{1}\right)=T^{t}\left(b_{2}\right)=0$, is classical. As usual, this is accomplished by subtracting from $T$ two paraproduct operators $\mathcal{L}, \mathcal{M}$ satisfying

$$
\mathcal{L}\left(b_{1}\right)=T\left(b_{1}\right), \mathcal{L}^{t}\left(b_{2}\right)=0 \text { and } \mathcal{M}^{t}\left(b_{2}\right)=T^{t}\left(b_{2}\right), \mathcal{M}\left(b_{1}\right)=0 .
$$


The existence of such operators is well known; cf., e.g., [DJS], [Me], [Da]. In the case when $b_{1}=b_{2}=1$, the operators $\mathcal{L}$ and $\mathcal{M}$ have a particularly simple form; see, e.g., [DJ], $[\mathbf{C h}]$. Re-denoting by $T$ the difference $T-\mathcal{L}-\mathcal{M}$, it follows that $T$ satisfies the same hypotheses as in Theorem 2.1 and, in addition, $T\left(b_{1}\right)=T^{t}\left(b_{2}\right)=0$.

Next, note that it suffices to show that $T$ maps $L^{2}\left(B_{R}(0)\right)$ boundedly into itself with norm controlled by the same constitutive constants as in the statement of Theorem 2.1, uniformly in $R$. That this implies the desired conclusion is easily seen by letting $R$ go to $\infty$ and using Fatou's lemma. Furthermore, it is enough to prove this only for $R=1$, i.e. for

$$
T: L^{2}\left(B_{1}(0)\right) \longrightarrow L^{2}\left(B_{1}(0)\right) \text {. }
$$

This is a manifestation of the dilation invariant nature of our assumptions. Specifically, if $\left(D_{\rho} f\right)(x):=f(\rho x)$ is the dilation operator of factor $\rho>0$, then $D_{R} T\left(D_{R^{-1}} f\right)=T_{R} f$ where $T_{R}$ is the operator associated with the kernel $R^{n} k(R x, R y)$. It is trivial to check that this kernel satisfies (2.1)-(2.2) with the same constant $\|k\|$. Also, $M_{D_{R} b_{2}} T_{R} M_{D_{R} b_{1}}$ satisfies the weak boundedness property with the same constant which works for $M_{b_{2}} T M_{b_{1}}$ and, since $D_{\rho}$ is an isometry of BMO for any $\rho>0,\left\|T_{R}\left(D_{R} b_{1}\right)\right\|_{\mathrm{BMO}}=\left\|T\left(b_{1}\right)\right\|_{\mathrm{BMO}}$, $\left\|T_{R}^{t}\left(D_{R} b_{2}\right)\right\|_{\text {BMO }}=\left\|T^{t}\left(b_{2}\right)\right\|_{\text {BMo }}$. Of course, $D_{R} b_{1}, D_{R} b_{2}$ remain bounded and accretive with the same bounds as $b_{1}, b_{2}$.

\section{Boundedness on Hölder spaces.}

With an eye toward proving the boundedness of the operator in (2.6) (with appropriate control) which suffices for our purposes, we shall now analyze the action of operators of this type on spaces of Hölder continuous functions. To state the main result in this direction recall that, for $0<\alpha<1$ and $\Omega \subseteq \mathbb{R}^{n}$,

$$
C^{\alpha}(\Omega):=\left\{f: \Omega \rightarrow \mathbb{C} ;\|f\|_{C^{\alpha}}:=\|f\|_{\infty}+\sup _{x, y \in \Omega}|x-y|^{-\alpha}|f(x)-f(y)|<\infty\right\} .
$$

Proposition 3.1. Let $T$ be a singular integral operator with kernel $k$ and let $b_{1}, b_{2}$ be two essentially bounded, accretive functions such that $M_{b_{2}} T M_{b_{1}}$ has the weak boundedness property and that $T\left(b_{1}\right)=0$. Then for any $\alpha \in(0,1)$ and any $\eta \in C_{\mathrm{c}}^{\infty}\left(B_{2}(0)\right)$ the operator

$$
M_{\eta} T M_{\eta}: b_{1} C^{\alpha}\left(B_{2}(0)\right) \longrightarrow C^{\alpha}\left(B_{2}(0)\right)
$$

is bounded with operator norm controlled in terms of $\alpha,\|k\|, \eta$, $\left\|M_{b_{2}} T M_{b_{1}}\right\|_{\mathrm{WBP}},\left\|b_{1}\right\|_{\infty},\left\|b_{2}\right\|_{\infty}$ and the accretivity constants.

The proof of this proposition involves only elementary estimates and closely related results have been known for a long time (compare with, e.g., 
[DJS], [Me]). However, for the reader's convenience, below we sketch a simple argument. To facilitate the presentation, we first isolate a technical result (which is precisely where the weak boundedness property is used).

Lemma 3.2. With the hypotheses of Proposition 3.1, there holds

$$
\left|\int_{|x-y|<\delta} k(x, y) b_{1}(y) d y\right| \leq C, \quad x \in \mathbb{R}^{n}
$$

uniformly for $0<\delta<\infty$, where $C$ is controlled by the same parameters as in the statement of Proposition 3.1.

Proof. To begin with, let us note that the integral in (3.3) may not converge absolutely and, in fact, must be interpreted in a distributional sense, as explained below.

Denote by $\chi_{E}$ the characteristic function of a set $E \subseteq \mathbb{R}^{n}$. Also, fix some even function $\psi \in C_{\mathrm{c}}^{\infty}\left(B_{3}(0)\right)$ with $\psi \equiv 1$ in $B_{2}(0)$ and set $\psi^{t, w}(x):=$ $\psi\left(\frac{x-w}{t}\right)$ for $t>0$ and $w \in \mathbb{R}^{n}$. Then, since $T\left(b_{1}\right)=0$, we may formally write

$$
\begin{aligned}
& \int_{|x-y|<\delta} k(x, y) b_{1}(y) d y \\
& =\int_{|x-y|>\delta}(k(x, y)-k(z, y)) b_{1}(y) d y \\
& \quad-T\left(b_{1} \psi^{\delta, x}\right)(z)-\int_{\mathbb{R}^{n}} k(z, y) b_{1}(y)\left(\chi_{B_{\delta}(x)}-\psi^{\delta, x}\right)(y) d y \\
& =: I_{1}(x, z)+I_{2}(x, z)+I_{3}(x, z) .
\end{aligned}
$$

Before going any further a comment is in order here. The point is that, generally speaking, the above integrals are to be understood in the sense of distributions. In particular, in order to derive size estimates, they should be integrated against (arbitrary) test functions. It is precisely in this sense that all our subsequent estimates must be interpreted even though, in order to shorten and simplify the exposition, we shall continue to manipulate such expressions in a formal manner. Of course, the main emphasis is to obtain bounds which depend exclusively on the relevant constants (as in the statement of Theorem 2.1).

Next, we shall average the identity (3.4). To this end, let $\phi \in C_{\mathrm{c}}^{\infty}\left(B_{1 / 2}(0)\right)$ be an even, nonnegative function with $\int_{\mathbb{R}^{n}} \phi d x=1$. Also, denote the argument of the complex number $\int_{|x-y|<\delta} k(x, y) b_{1}(y) d y$ by $\theta=\theta(x, \delta) \in[0,2 \pi)$. 
Then, by virtue of the accretivity of $b_{2}$,

$$
\begin{aligned}
& C\left|\int_{|x-y|<\delta} k(x, y) b_{1}(y) d y\right| \\
& \leq \operatorname{Re} \int_{\mathbb{R}^{n}} b_{2}(z) \delta^{-n} \phi^{\delta, x}(z) e^{i \theta}\left(\int_{|x-y|<\delta} k(x, y) b_{1}(y) d y\right) d z \\
& =\sum_{j=1}^{3} \operatorname{Re} \int_{\mathbb{R}^{n}} b_{2}(z) \delta^{-n} \phi\left(\frac{x-z}{\delta}\right) e^{i \theta} I_{j}(x, z) d z \\
& =: I I_{1}(x)+I I_{2}(x)+I I_{3}(x) .
\end{aligned}
$$

For $I I_{1}(x)$, the mean value theorem, (2.2) and the fact that $|x-z| \leq \frac{1}{2}|x-y|$ on the domain of integration give

$$
\left|I I_{1}(x)\right| \leq C\|k\|\left\|b_{1}\right\|_{\infty}\left\|b_{2}\right\|_{\infty} \delta^{-n} \int_{\mathbb{R}^{n}} \phi\left(\frac{x-z}{\delta}\right) \int_{\delta<|x-y|} \frac{|x-z|}{|x-y|^{n+1}} d y d z \leq C .
$$

Also, $I I_{2}(x)$ is clearly controlled by $\left\|M_{b_{2}} T M_{b_{1}}\right\|_{\text {WBP }}$, whereas

$$
\left|I I_{3}(x)\right| \leq C\left\|b_{1}\right\|_{\infty}\left\|b_{2}\right\|_{\infty} \int_{\mathbb{R}^{n}} \delta^{-n} \phi\left(\frac{x-z}{\delta}\right)\left(\int_{\delta / 2<|z-y|<7 \delta / 2}|k(z, y)| d y\right) d z .
$$

Since $k$ is standard, the last inner integral is trivially bounded independently of $\delta$ and $z$ and the desired conclusion follows.

Based on this result it is now easy to tackle the

Proof of Proposition 3.1. Fix some $0<\alpha<1$ and take an arbitrary $f \in C^{\alpha}\left(B_{2}(0)\right)$. Then, for $x \in B_{2}(0)$ we write

$$
\begin{aligned}
T\left(b_{1} \eta f\right)(x)= & \int_{|x-y| \leq 4} k(x, y) b_{1}(y)((\eta f)(y)-(\eta f)(x)) d y \\
& +(\eta f)(x) \int_{|x-y| \leq 4} k(x, y) b_{1}(y) d y .
\end{aligned}
$$

In particular,

$$
\left\|T\left(M_{\eta} b_{1} f\right)\right\|_{L^{\infty}\left(B_{2}(0)\right)} \leq C\|f\|_{C^{\alpha}\left(B_{2}(0)\right)} .
$$

Next, for $x, h \in \mathbb{R}^{n}$ with $|x|,|x+h| \leq 2$, we may write on account of the fact that $T\left(b_{1}\right)=0$

$$
\left|T\left(b_{1} \eta f\right)(x+h)-T\left(b_{1} \eta f\right)(x)\right| \leq I+I I+I I I,
$$


where

$$
\begin{aligned}
I & :=\left|\int_{|x-y| \geq 2|h|}(k(x+h, y)-k(x, y)) b_{1}(y)((\eta f)(y)-(\eta f)(x)) d y\right|, \\
I I & :=\left|\int_{|x-y| \leq 2|h|} k(x, y) b_{1}(y)((\eta f)(y)-(\eta f)(x)) d y\right|, \\
I I I & :=\left|\int_{|x-y| \leq 2|h|} k(x+h, y) b_{1}(y)((\eta f)(y)-(\eta f)(x)) d y\right| .
\end{aligned}
$$

We seek a bound of the order of $|h|^{\alpha}\|f\|_{C^{\alpha}\left(B_{2}(0)\right)}$ for each quantity above. Based solely on (2.1)-(2.2), the first two integrals are handled in a crude, straightforward fashion and we omit the details. It is only the last term which requires one more application of Lemma 3.2. Indeed, we have

$$
\begin{aligned}
|I I I| \leq & \int_{|x-y| \leq 2|h|}\left|k(x+h, y) b_{1}(y)((\eta f)(y)-(\eta f)(x+h))\right| d y \\
& +|h|^{\alpha}\|\eta f\|_{C^{\alpha}\left(B_{2}(0)\right)}\left|\int_{|x-y| \leq 2|h|} k(x+h, y) b_{1}(y) d y\right| \\
= & : I I I_{1}+|h|^{\alpha}\|\eta f\|_{C^{\alpha}\left(B_{2}(0)\right)} I I I_{2} .
\end{aligned}
$$

The first integral, $I I I_{1}$, is treated essentially as $I I$ above, producing a bound of the same order. Finally, for the second integral, Lemma 3.2 and (2.1) give

$$
\begin{aligned}
\left|I I I_{2}\right| & \leq\left|\int_{|x+h-y|<|h|} \ldots\right|+\int_{|h|<|x+h-y|<3|h|}|\ldots| \\
& \leq C_{0}+C_{1}\left\|b_{1}\right\|_{\infty}\|k\| \int_{|h|<|z-y|<3|h|} \frac{d y}{|z-y|^{n}} d y \leq C_{2} .
\end{aligned}
$$

This yields the right estimate for $I I I$ also. To sum up, we have proved that

$$
\sup _{x, y \in \mathbb{R}^{n}}|x-y|^{-\alpha}\left|T\left(M_{\eta} b_{1} f\right)(x)-T\left(M_{\eta} b_{1} f\right)(y)\right| \leq C\|f\|_{C^{\alpha}\left(B_{2}(0)\right)}
$$

which, together with (3.8), readily implies the desired conclusion.

\section{A functional analysis lemma and the end of the proof of Theorem 2.1.}

What allows us to pass from continuity on spaces of Hölder continuous functions to continuity on spaces of square integrable functions is a certain functional analytic argument which is a variation of a lemma due to M.G. Krein $[\mathbf{K r}]$ (cf. also [La], [Di]). 
Lemma 4.1. Let $\mathcal{X}$ be a Banach space and assume that

$$
(\cdot, \cdot)_{i}: \mathcal{X} \times \mathcal{X} \longrightarrow \mathbb{C}, \quad i=1,2,
$$

are two forms such that, for some $M>0$,

$$
\left|(x, y)_{i}\right| \leq M\|x\|_{\mathcal{X}}\|y\|_{\mathcal{X}} \quad \forall x, y \in \mathcal{X}, \quad i=1,2 .
$$

We also assume that each $(\cdot, \cdot)_{i}, i=1,2$, is accretive and symmetric in the sense that

$$
\operatorname{Re}(x, x)_{i} \geq \kappa\|x\|_{\mathcal{X}}^{2}, \quad \text { and } \quad(x, y)_{i}=\overline{(y, x)_{i}}, \quad \forall x, y \in \mathcal{X} .
$$

Consider next a linear normed space $\mathcal{Y}$ for which the inclusion $\iota: \mathcal{Y} \hookrightarrow \mathcal{X}$ is well defined, continuous and with dense range. Finally, let $A, B: \mathcal{Y} \longrightarrow \mathcal{Y}$ be two bounded, linear operators so that

$$
(A x, y)_{1}=(x, B y)_{2}, \quad \forall x, y \in \mathcal{Y} .
$$

Then both $A$ and $B$ extend to continuous operators on $\mathcal{X}$ with

$$
\|A\|_{\mathcal{X} \rightarrow \mathcal{X}},\|B\|_{\mathcal{X} \rightarrow \mathcal{X}} \leq M \kappa^{-1}\|A\|_{\mathcal{Y} \rightarrow \mathcal{Y}}^{1 / 2}\|B\|_{\mathcal{Y} \rightarrow \mathcal{Y}}^{1 / 2} .
$$

Proof. For an arbitrary $x \in \mathcal{Y}$, using (4.2)-(4.4) it follows that

$$
\kappa\|A x\|_{\mathcal{X}}^{2} \leq\left|(A x, A x)_{1}\right|=\left|(x, B A x)_{2}\right| \leq M\|x\|_{\mathcal{X}}\|B A x\|_{\mathcal{X}} .
$$

Therefore,

$$
\|A x\|_{\mathcal{X}} \leq \kappa^{-1 / 2} M^{1 / 2}\|x\|_{\mathcal{X}}^{1 / 2}\|B A x\|_{\mathcal{X}}^{1 / 2}
$$

which, in particular, shows that it suffices to prove that $B A$ extends to a continuous operator on $\mathcal{X}$ with a suitable bound for its norm. To this end, paralleling (4.6)-(4.7) but with $B A$ in place of $A$ (note that $B A$ has a bounded transpose in the sense of (4.4)) gives the estimate $\|(B A) x\|_{\mathcal{X}} \leq$ $\kappa^{-1 / 2} M^{1 / 2}\|x\|_{\mathcal{X}}^{1 / 2}\left\|(B A)^{2} x\right\|_{\mathcal{X}}^{1 / 2}$. The key observation is that, in fact, any power $(B A)^{N}$ will work in place of $B A$ in this last inequality and, hence, iterating $j$ times according to powers of 2 gives

$$
\begin{aligned}
& \|(B A) x\|_{\mathcal{X}} \\
& \leq(M / \kappa)^{2^{-1}+\cdots+2^{-j}}\|x\|_{\mathcal{X}}^{2^{-1}+\cdots+2^{-j}}\left\|(B A)^{2^{j}} x\right\|_{\mathcal{X}}^{2^{-j}} \\
& \leq(M / \kappa)^{2^{-1}+\cdots+2^{-j}}\|x\|_{\mathcal{X}}^{2^{-1}+\cdots+2^{-j}}\|\iota\|_{\mathcal{Y} \rightarrow \mathcal{X}}^{2^{-j}}\left(\left\|(B A)^{2^{j}}\right\| \mathcal{Y} \rightarrow \mathcal{Y}\right)^{2^{-j}}\|x\|_{\mathcal{Y}}^{2^{-j}} \\
& \leq(M / \kappa)^{2^{-1}+\cdots+2^{-j}}\|x\|_{\mathcal{X}}^{2^{-1}+\cdots+2^{-j}}\|\iota\|_{\mathcal{Y} \rightarrow \mathcal{X}}^{2^{-j}}\|B A\|_{\mathcal{Y} \rightarrow \mathcal{Y}}\|x\|_{\mathcal{Y}}^{2^{-j}} .
\end{aligned}
$$

Passing to the limit $j \rightarrow \infty$ yields $\|(B A) x\|_{\mathcal{X}} \leq M \kappa^{-1}\|x\|_{\mathcal{X}}\|B A\|_{\mathcal{Y} \rightarrow \mathcal{Y}}$ as desired. 
Parenthetically, let us note that one can avoid arbitrarily high order iterations in the above reasoning. Indeed, for each $x \in \mathcal{Y}$ with $\|x\|_{\mathcal{X}} \leq 1$, set

$K(x):=\sup \left\{\|A x\|_{\mathcal{X}} ; \forall A, B\right.$ which satisfy the hypotheses of the lemma

$$
\text { and } \left.\|A\|_{\mathcal{Y} \rightarrow \mathcal{Y}},\|B\|_{\mathcal{Y} \rightarrow \mathcal{Y}} \leq 1\right\} \text {. }
$$

Now, by (4.7) it follows that $K(x) \leq \kappa^{-1 / 2} M^{1 / 2} K(x)^{1 / 2}$ and, further, $K(x) \leq \kappa^{-1} M$, since $K(x)$ is finite. The estimate (4.5) is then obtained by rescaling.

With all ingredients in place, we are now in a position to present the final details in the proof of the Theorem 2.1. To this effect, let us recall that we have reduced matters to showing that the operator $T$ in (2.6) is bounded with operator norm appropriately controlled. Further, it is trivial to check that this is true if we can show that, for a fixed real valued function $\eta \in C_{\mathrm{c}}^{\infty}\left(B_{2}(0)\right)$ with $\eta \equiv 1$ on $B_{1}(0)$,

$$
M_{\eta} T M_{\eta}: L^{2}\left(B_{2}(0)\right) \longrightarrow L^{2}\left(B_{2}(0)\right)
$$

is bounded with the right norm control. The important thing is that $T\left(b_{1}\right)=$ $T^{t}\left(b_{2}\right)=0$ so that Proposition 3.1 applied both to $T$ and $T^{t}$ gives that

$$
\begin{array}{r}
M_{\eta} T M_{\eta}: b_{1} C^{\alpha}\left(B_{2}(0)\right) \longrightarrow C^{\alpha}\left(B_{2}(0)\right) \\
M_{\eta} T^{t} M_{\eta}: b_{2} C^{\alpha}\left(B_{2}(0)\right) \longrightarrow C^{\alpha}\left(B_{2}(0)\right)
\end{array}
$$

are bounded with appropriate control.

At this point Lemma 4.1 applies to lift this to the $L^{2}$ setting in the following context. We take $\mathcal{X}:=L^{2}\left(B_{2}(0)\right), \mathcal{Y}:=C^{\alpha}\left(B_{2}(0)\right), A:=M_{\eta} T M_{\eta} M_{b_{1}}$, $B:=\overline{M_{\eta} T^{t} M_{\eta} M_{b_{2}}}$, where, if $U$ is an operator then $\bar{U} f:=\overline{U \bar{f}}$, and

$$
(f, g)_{1}:=\int f b_{2} \bar{g} d x, \quad(f, g)_{2}:=\int f b_{1} \bar{g} d x, \quad f, g \in L^{2}\left(B_{2}(0)\right) .
$$

Since $b_{1}$ and $b_{2}$ are real-valued we have that $(f, g)_{i}=\overline{(g, f)_{i}}$ for $i=1,2$. Next, the accretivity of $b_{i}$ entails the accretivity of the corresponding paring $(\cdot, \cdot)_{i}, i=1,2$, whereas

$$
\begin{aligned}
(A f, g)_{1} & =\int M_{\eta} T M_{\eta} M_{b_{1}} f M_{b_{2}} \bar{g} d x=\int f M_{b_{1}} M_{\eta} T^{t} M_{\eta} M_{b_{2}} \bar{g} d x \\
& =\int f M_{b_{1}} \overline{\overline{M_{\eta} T^{t} M_{\eta} M_{b_{2}}}} d x=(f, B g)_{2} .
\end{aligned}
$$

Note that the third equality above follows from the fact that $U \bar{f}=\overline{\bar{U} f}$. Thus, since $M_{b_{1}}$ is a isomorphism of $L^{2}\left(B_{2}(0)\right)$ onto itself, Lemma 4.1 gives that $M_{\eta} T M_{\eta}$ is bounded on $L^{2}\left(B_{2}(0)\right)$ with the desired norm control. The proof of the Theorem 2.1 is therefore finished. 


\section{References}

[Ca] A. Calderón, Cauchy integrals on Lipschitz curves and related operators, Proc. NAS USA, 74 (1977), 1324-1327.

[Ch] M. Christ, Lectures on Singular Integral Operators, CBMS No. 77, AMS 1990.

[CDM] R. Coifman, G. David and Y. Meyer, La solution des conjectures de Calderón, Adv. in Math, 48 (1983), 144-148.

[CJS] R. Coifman, P.W. Jones and S. Semmes, Two elementary proofs of the $L^{2}$ boundedness of the Cauchy integral on Lipschitz curves, Jour. of Amer. Math. Soc., 2 (1989), 553-564.

[CMM] R. Coifman, A. McIntosh and Y. Meyer, L'intégrale de Cauchy definit un opérateur borné sur $L^{2}$ pour les courbes Lipschitziennes, Annals of Math., 116 (1982), 361-388.

[CoWe] R. Coifman and G. Weiss, Extensions of Hardy spaces and their use in analysis, Bull. Amer. Math. Soc., 83 (1977), 569-645.

[Da] G. David, Wavelets and Singular Integrals on Curves and Surfaces, Springer Verlag LNM No. 1465, 1994.

[DJ] G. David and J.-L. Journé, A boundedness criterion for generalized CalderónZygmund operators, Ann. Math., 120 (1984), 371-397.

[DJS] G. David, J.-L. Journé and S. Semmes, Opérateurs de Calderón-Zygmund, fonctions para-accrétives, et interpolation, Rev. Mat. Iberoamericana, 1 (1985), 1-56.

[DJS2] — Calderón-Zygmund operators, para-accretive functions and interpolation, Preprint.

[Di] J.A. Dieudonné, Quasi-hermitian operators, in 'Proceedings of the International Symposium on Linear Spaces', Jerusalem, (1996) 115-122.

[Kr] M.G. Krein, On linear continuous operators in functional spaces with two norms, Trudy Inst. Mat. Akad. Nauk Ukrain. SSRS, 9 (1947), 104-129.

[La] P.D. Lax, Symmetrizable linear transformations, Comm. Pure Appl. Math., 7 (1954), 633-647.

[MM] A. McIntosh and Y. Meyer, Algebres d'opérateurs definies par des integrales singulieres, C. R. Acad. Sci. Paris, Ser. I Math., 301 (1985), 395-397.

[Me] Y. Meyer, Ondelettes et Opérateurs, II, Hermann, Paris, 1990.

[St] E. Stein, Harmonic Analysis: Real-Variable Methods, Orthogonality, and Oscillatory Integrals, Princeton Mathematical Series 43, Princeton University Press, Princeton, NJ, 1995.

Received August 15, 1997 and revised July 14, 1998. The first author was partially supported by a UMC Research Board grant and a NSF grant.

University of Minnesota

MinNEAPOLIS, MN 55455

UNIVERSity OF Missouri

Columbia, MO 65211

University of Missouri

Columbia, MO 65211

E-mail address: marius@math.missouri.edu 